\title{
Aprendizagem Cooperativa e a Formação do Médico Inserido em Metodologias Ativas: um Olhar de Estudantes e Docentes
}

\author{
Cooperative Learning and the Training of the \\ Physicians through Active Methodologies: Views \\ of Students and Teachers
}

Caio Vinícius da Conceição ${ }^{I}$ Magali Aparecida Alves de Moraes ${ }^{I}$

\author{
PALAVRAS-CHAVE \\ - Educação Médica. \\ - Aprendizagem Baseada em \\ Problemas. \\ - Educação Continuada. \\ - Comportamento Cooperativo. \\ - Ensino.
}

\begin{abstract}
RESUMO
INTRODUÇÃO: Com a finalidade de adquiriras competências médicas preconizadas pelas Diretrizes Curriculares Nacionais, as instituições acadêmicas da área da saúde têm buscado novas estratégias. Nessa conjuntura, observa-se que a aprendizagem cooperativa, ao ser estimuladano âmbito de metodologias ativas de ensino e aprendizagem, pode trazer diferenciais importantes na formação do médico. Assim, o objetivo geral deste estudo foi explorar a percepção de estudantes e docentes sobre aprendizagem cooperativa em um currículo médico baseado em métodos ativos (Aprendizagem Baseada em Problemas e Problematização). Os objetivos específicos foram investigar os benefícios da cooperação no trabalho em pequenos grupos de estudantes e identificar as necessidades de adequação dos docentes em sua atuação nesses grupos reduzidos. MÉTODOS: O estudo é descritivo, qualitativo e quantitativo, com aplicação de questionários e entrevistas semiestruturadas a estudantes da primeira à quarta série de Medicina e docentes participantes de tutorias e ciclos pedagógicos. Os dados provenientes dos questionários foram dispostos em tabelas e avaliados por meio da escala do tipo Likert. As entrevistas foram gravadas e transcritas para posterior realização de agrupamento de núcleos de sentido para análise de conteúdo, modalidade temática. A pesquisa foi conduzida com fomento e apoio do Pibic/CNPQ com o processo de no 115007/2016-4. RESULTADOS E DISCUSSÃO: No que se refere ao perfil dos estudantes, a maioria se situava entre 22 e 25 anos, majoritariamente do sexo feminino e de cor branca. Em relação aos docentes, $85 \%$ se graduaram em método tradicional,tendo de 16 a 30 anos de formação e de 15 a 21 anos de trabalho com métodos ativos, sendo que 92\% deles com pós-graduação. Os resultados quantitativos e qualitativos foram convergentes, estudantes e docentes apresentaram concepções análogas quanto aos benefícios da aprendizagem cooperativa em pequenos grupos, julgando que habilidades de comunicação, raciocínio crítico, interdependência positiva, avanços em trabalho em equipe, além de boa aquisição de conhecimento cognitivo são garantidos nesse processo. Observou-se também que, à medida que avançavam nas séries, os estudantes expressavam percepções um pouco diferentes em relação a diversas questões, o que pode estar relacionado à maturidade que advém com o passar dos anos e principalmente à experiência com os métodos ativos. CONSIDERAÇÕES FINAIS: A aprendizagem cooperativa é eficaz em pequenos grupos, traz benefícios sociais, cognitivos e psicomotores, ée favorecida no âmbito de metodologias ativas com educação permanente dos professores. Os resultados encontrados neste estudo poderiam ser úteis para outras faculdades de Medicina que almejam trabalhar com métodos ativos ou mesmo para as que já os utilizam com a finalidade de orientar gestores e coordenadores do curso.
\end{abstract}




\section{KEY-WORDS}

- Medical Education.

- Problem-Based Learning.

- Continuing Education.

- Cooperative Behaviour.

- Teaching.

\begin{abstract}
INTRODUCTION: In order to acquire the medical skills recommended by the National Curricular Guidelines, academic institutions in the area of heath have looked for new strategies. In this context, it is seen that cooperative learning, when stimulated in the framework of active teaching-learning methodologies, can bring important advantages for medical training. The general objective of this study was to explore students' and teachers' views on cooperative learning in a medical curriculum based on active methods (Problem Based-Learning and Investigation). The specific objectives were: to investigate the benefits of cooperation in small groups of students, and to identify the needs for teachers to adapt their practices when working with these small groups. METHODS: This is a descriptive, quantitative and qualitative study, with the application of questionnaires and semi structured interviews to students in the first to fourth years of medical school, and teachers participating in tutorials and pedagogical cycles. The data from the questionnaires were arranged in tables and evaluated through a Likert scale. The interviews were recorded and transcribed for subsequent grouping of cores of meaning for content analysis, thematic modality. The research was supported by Pibic/CNP, with process number 115007/2016-4. RESULTS AND DISCUSSION: Regarding the student's profile, the majority were aged between 22 and 25 years, female, and White. Regarding the teachers' profile, $85 \%$ had graduated by the traditional method, with 16 to 30 years since graduating,15 to 21 years of working with active methods, and 92\% with postgraduate degrees. The quantitative and qualitative results were convergent; the students and teachers presented analogous conceptions about the benefits ofcooperative learning in small groups, believing that communication skills, critical thinking, positive interdependence, advance toward teamwork, and the acquisition of cognitive knowledge are guaranteed in this process. It was also observed that as the students moved up through the grades, their views changed slightly in relation to several questions. This may be realted to the extra maturity that comes with the passage of years, but it is largely due to the experience withactive methods. FINALCONSIDERATIONS: Cooperative learning is efficient within small groups. It brings social, cognitive and psychomotor benefits, and it is favored in the area of active methodologies with lifelong learning. The results of this study could be useful for providing guidance to managers and coordinators of other medical courses seeking to use active methodologies, or even for those that already use them.
\end{abstract}

\section{INTRODUÇÃO}

Em consonância com as Diretrizes Curriculares Nacionais do Curso de Graduação em Medicina (DCN) ${ }^{1}$, o egresso do curso médico, além de ter uma formação geral e atuar com promoção e prevenção à saúde em âmbito individual e coletivo, deverá ser humanizado e exercer suas funções de acordo com princípios éticos e responsabilidade social, com a finalidade de reconhecer o indivíduo em toda a sua singularidade e cidadania, estando capacitado a identificar as diferentes dimensões inerentes ao indivíduo e comunidade, e considerando o espectro da diversidade humana das esferas cultural, étnico-racial e sexual.

A fim de prover assistência de qualidade de acordo com as exigências do sistema de saúde, o profissional deve criar, implementar e planejar políticas intervencionistas. Na expectativa de atingir este objetivo, as instituições acadêmicas da área da saúde têm investido esforços a fim de buscar novas estratégias para a aquisição dessas competências médicas. Nessa conjuntura, observa-se que a cooperação, ao ser estimulada no domínio de metodologias ativas de ensino e aprendizagem, pode trazer diferenciais importantes na formação do médico, potencializando o aprendizado individual e coletivo ${ }^{2}$.

Metodologias ativas de ensino e aprendizagem têm sido adotadas a fim de privilegiar a participação ativa do estudante, levando-o a ser protagonista na construção do seu conhecimento, propiciando maior cooperação entre os discentes e maximizando a interação entre ensino, assistência e pesquisa. A Aprendizagem Baseada em Problemas (ABP) é a metodo- 
logia mais utilizada em cursos médicos brasileiros que promovem a aprendizagem cooperativa com foco na construção do conhecimento de forma ativa, preparando o futuro médico para exercer sua profissão de acordo com as necessidades do sistema de saúde ${ }^{3}$.

Para que se compreenda a aplicabilidade das metodologias expostas, é interessante notar que há um individualismo imperando na sociedade, o qual impele os cidadãos a serem menos colaborativos com o grupo, o que prejudica as relações sociais e a execução de trabalhos coletivos ${ }^{4}$. Em contrapartida à filosofia vigente na década de 1970, incentivou-se a estruturação e utilização da aprendizagem cooperativa como um recurso que fomenta rendimento acadêmico, interdependência positiva, responsabilidade individual e de grupo, interação estimuladora, competências sociais, além de aumento de autoestima ${ }^{5}$.

Para melhor elucidação conceitual, é necessário caracterizar o entendimento de Piaget acerca de cooperação. Segundo esse autor, interações interindividuais, fomentadas por formação de vínculos e reciprocidade afetiva, possibilitam modificar o sujeito em sua estrutura cognitiva, propiciando a construção do conhecimento ${ }^{6}$. Dessa forma, aprendizagem cooperativa é definida a partir do trabalho de estudantes em pequenos grupos heterogêneos que trocam informações e compartilham materiais, com papéis previamente definidos, conscientes de que só alcançarão sucesso se todos os elementos do grupo também o obtiverem ${ }^{7}$.

Nesse contexto, os docentes que atuam em instituições contempladas com as reformulações curriculares devem estar adaptados ao novo modelo, planejando execuções coerentes com a proposta solicitada. Estudos enfatizam a relativa dificuldade da docência médica e universal no domínio de seu desempenho com a utilização da ABP e a consequente disposição de estudantes em pequenos grupos cooperativos, uma vez que os papéis de ativador e mediador dos tutores devem ser pronunciados na busca de soluções para os problemas identificados por eles ${ }^{8}$.

Assim, o presente estudo teve como objetivo geral explorar a percepção de estudantes e docentes sobre aprendizagem cooperativa em um currículo médico baseado em métodos ativos; e como objetivos específicos: investigar os benefícios da cooperação no trabalho em pequenos grupos de estudantes em âmbito de metodologias ativase identificar as necessidades de adequação dos docentes no que se refere a sua atuação empequenos grupos cooperativos de estudantes.

\section{MÉTODO}

Trata-se de um estudo descritivo, quantitativo e qualitativo, no qual a coleta de dados ocorreu de setembro de 2016 a maio de 2017. Na modalidade quantitativa, caracterizou-se o perfil de estudantes e docentes da Faculdade de Medicina de Marília (Famema) quanto a suas percepções no que tange à aprendizagem cooperativa instigada na esfera de metodologias ativas de ensino e aprendizagem (ABP e Problematização) em pequenos grupos na graduação. Neste módulo do estudo, também foram identificados aspectos sobre a capacitação e adequação dos docentes neste processo. O universo dos participantes da pesquisa envolveu estudantes da primeira à quarta série matriculados no curso de Medicina da Famema e docentes atuantes em atividades de tutorias e ciclos pedagógicos desses mesmos anos. Assim, para que estes pudessem ser reconhecidos, solicitou-se à secretaria-geral da faculdade uma listagem com os nomes de todos os professores que tivessem atribuições com alunos do primeiro ao quarto ano da instituição, bem como a listagem dos estudantes por série. A partir daí, a seleção dos participantes da pesquisa se deu de modo aleatório.

A coleta de dados nessa etapa da pesquisa foi realizada por intermédio de dois questionários distintos com escalas do tipo Likert, um destinado a estudantes e outro a docentes. A parte inicial dos questionários continha dados referentes ao perfil de ambos, e a seguinte continha todas as demais questões. As amostras foram definidas assumindo-se um nível de significância de 5\%, intervalo de confiança de 95\% e heterogeneidade de 50\% dentro do Cálculo de Amostragem Normal de Bolfarine e Bussab 9 . Assim, foram selecionados aleatoriamente $60 \%$ dos estudantes,perfazendo 48 estudantes por série de um total de 320 . No que se refere aos docentes, 75,2\% destes foram elegidos,totalizando 97 de 129. Ao término da coleta dos dados, estes foram dispostos em planilhas de Excel 2010 for Windows para melhor adequar as informações e visualizar os resultados.

Na modalidade qualitativa, investigou-se o conhecimento dos estudantes e docentes sobre aprendizagem cooperativa e seus benefícios, bem como se esta ferramenta estava sendo eficaz na maximização da aprendizagem significativa individual e em grupo nas atividades da graduação. Nesta seção, foram utilizadas duas entrevistas semiestruturadas diferentes, uma direcionada a estudantes e outra a professores. Da mesma forma que os questionários, continham questões iniciais relativas ao perfil dos entrevistados, seguindo com questões abertas a respeito dos temas da pesquisa. Estes instrumentos foram aplicados até que houvesse repetição do conteúdo das falas, o que ocorreu após oito entrevistas para estudantes (duas por 
série) e sete entrevistas para docentes, os quais eram médicos, enfermeiros, profissionais das cadeiras básicas e coordenadores do curso ${ }^{10}$.

Para posterior análise, as entrevistas foram gravadas e transcritas integralmente, excluindo-se apenas vícios de linguagem para melhor entendimento do texto, que em nada alteravam a fidedignidade dos discursos. No intuito de garantir o anonimato, as falas dos participantes docentes foram transcritas com a letra " $\mathrm{P}$ ", seguida do número para análise, e a letra "E"foi usada para os estudantes. Os dados provenientes das entrevistas foram analisados, categorizados em núcleos de sentido e agrupados posteriormente em categorias temáticas, com a técnica de análise de conteúdo, modalidade temática ${ }^{11,12}$.

A fim de orientar os participantes da pesquisa e obter sua permissão para obter os dados provenientes dos questionários e das entrevistas, foi apresentado o Termo de Consentimento Livre e Esclarecido aos participantes em ambos os momentos, os quais foram assinados para que os processos se iniciassem. O projeto foi aprovado pelo Comitê de Ética em Pesquisa da Famema em 06/07/2016 por meio do Parecer Consubstanciado $n^{-1}$ 1.624.400, de acordo com a Resolução CNS/MS nº 466/2012 para autorização de seu desenvolvimento. Além disso, este artigo faz parte da pesquisa que foi conduzida com fomento e apoio do Pibic/CNPQ com o n⿳o do processo 115007/2016-4 e apresentada oralmente à banca examinadora em evento promovido pela instituição.

\section{RESULTADOS E DISCUSSÃO}

A Tabela 1 expressa o perfil dos estudantes da primeira à quarta série da Famema por sexo, cor e idade. Assim, percebe-se que a maioria se situava entre 22 e 25 anos, de cor branca e majoritariamente do sexo feminino, o que reflete a tendência atual de aumento do preenchimento de vagas de Medicina por mulheres. A ocupação e o estado civil dos participantes também foram avaliados, com a quase unanimidade destes afirmando ser estudante sua única ocupação (96\% a 98\% entre as séries) e $100 \%$ de solteiros.

As questões seguintes elucidaram as percepções dos estudantes que trabalham em pequenos grupos acerca de aprendizagem cooperativa no âmbito de metodologias ativas de ensino e aprendizagem. Ao serem indagados se havia contribuição significativa dos outros estudantes em sua aprendizagem nos grupos tutoriais e ciclos pedagógicos, a maioria nas quatro séries concordou com esta afirmação, sendo $65 \%$ na primeira; $75 \%$ na segunda; $63 \%$ na terceira; e $79 \%$ na quarta série.

Ao se deparar com a proposição que os colocava diante do papel do tutor em sua aprendizagem dentro das tutorias, a maioria dos estudantes da primeira série (75\%) afirmou que

\begin{tabular}{|c|c|c|c|c|c|c|c|c|}
\hline $\begin{array}{r}\text { Cara } \\
\text { Me }\end{array}$ & $\begin{array}{l}\text { teri } \\
\text { dici }\end{array}$ & $\begin{array}{l}\text { ăo } \\
\text { la } 1\end{array}$ & $\begin{array}{l}\text { iod } \\
\text { mei }\end{array}$ & $\begin{array}{l}\text { ELA } \\
\text { ogr } \\
\text { qu }\end{array}$ & a c & $\begin{array}{l}\text { est } \\
\text { da }\end{array}$ & & \\
\hline Variáveis & & & & & & & & \\
\hline $\begin{array}{l}\text { Sexo } \\
\text { F } \\
\text { M }\end{array}$ & $\begin{array}{l}26 \\
22\end{array}$ & $\begin{array}{l}54 \\
46\end{array}$ & $\begin{array}{l}32 \\
16\end{array}$ & $\begin{array}{l}67 \\
33\end{array}$ & $\begin{array}{l}26 \\
22\end{array}$ & $\begin{array}{l}54 \\
46\end{array}$ & $\begin{array}{l}29 \\
19\end{array}$ & $\begin{array}{l}60 \\
40\end{array}$ \\
\hline $\begin{array}{l}\text { Cor } \\
\text { Branca } \\
\text { Negra } \\
\text { Parda } \\
\text { Amarela }\end{array}$ & $\begin{array}{c}41 \\
0 \\
1 \\
6\end{array}$ & $\begin{array}{c}85 \\
0 \\
2 \\
13\end{array}$ & $\begin{array}{c}35 \\
2 \\
3 \\
8\end{array}$ & $\begin{array}{c}73 \\
4 \\
6 \\
17\end{array}$ & $\begin{array}{c}40 \\
0 \\
3 \\
5\end{array}$ & $\begin{array}{c}83 \\
0 \\
6 \\
11\end{array}$ & $\begin{array}{c}37 \\
1 \\
0 \\
10\end{array}$ & $\begin{array}{c}77 \\
2 \\
0 \\
21\end{array}$ \\
\hline $\begin{array}{l}\text { Idade } \\
18-21 \\
22-25 \\
26-29\end{array}$ & $\begin{array}{c}38 \\
9 \\
1\end{array}$ & $\begin{array}{c}79 \\
19 \\
2\end{array}$ & $\begin{array}{c}19 \\
24 \\
5\end{array}$ & $\begin{array}{l}40 \\
50 \\
10\end{array}$ & $\begin{array}{c}13 \\
31 \\
4\end{array}$ & $\begin{array}{c}27 \\
65 \\
8\end{array}$ & $\begin{array}{c}1 \\
41 \\
6\end{array}$ & $\begin{array}{c}2 \\
94 \\
4\end{array}$ \\
\hline Total & $N=$ & $6=$ & & & & & & \\
\hline
\end{tabular}

considera preponderante o papel do tutor no processo tutorial. Na segunda série, $90 \%$ consideraram essencial. A terceira série teve a menor porcentagem de concordância (54\%), enquanto $71 \%$ da quarta série concordaram. Assim, percebe-se que há valorização do papel do tutor/facilitador.

De acordo com alguns autores ${ }^{13}$, o tutor poderia ser definido como aquele profissional que ensina o estudante a aprender, sendo essencial num pequeno grupo pela sua experiência ao aconselhar, guiar e facilitar a construção do conhecimento. Portanto, ao tornar-se modelo, seu principal papel remete ao âmbito profissional. Porém, apesar de não mandatório, este também pode prover aos estudantes suporte na esfera pessoal, auxiliando-os na adaptação à nova vivência. Desta forma, ao reconhecerem a importância do tutor no processo tutorial, os estudantes desta pesquisa corroboram as expectativas da literatura médica acerca desse profissional.

Quando investigado se consideravam que avaliações formativas realizadas ao fim de cada processo tutorial ou dos ciclos pedagógicos propiciavam melhor autocrítica, melhoria da aprendizagem, aperfeiçoamento em habilidades de comunicação, trabalho em equipe e relações interpessoais, a maioria dos estudantes de todas as séries concordou com isso: na primeira, 69\%; na segunda, 69\%; na terceira, 54\%; e na quarta, $54 \%$. Embora o percentual decresça à medida que a série avança, percebe-se que há valorização desse movimento avaliativo.

Reforçando os benefícios advindos com as avaliações formativas em pequenos grupos durante as atividades da graduação, num estudo de Costa et al. ${ }^{14}$, professores e estudantes reconhecem o benefício da cooperação mútua em atividades de simulações de situações clínicas relevantes no Laboratório 
de Prática Profissional; o feedback imediato por todos os elementos do grupo contribui com o desenvolvimento da aprendizagem de todos.

Quanto à proposição segundo a qual no pequeno grupo percebiam mais cooperação do que competição, na primeira série, 37\% concordaram;na segunda série, 54\% concordaram;na terceira série, $61 \%$ concordaram;e na quarta série, $67 \%$ concordaram. Parece que no primeiro ano ainda trazem fortementeem suas atitudes a competição, talvez porque tenham acabado de sair do vestibular, e, portanto, a experiência com os métodos facilita a cooperação entre eles. Segundo meta-análise de Albanese $^{15}$ realizada para avaliar os efeitos da aprendizagem cooperativa em comparação com a aprendizagem competitiva, ações cooperativas trouxeram maiores benefícios na resolução de problemas $(72,5 \%)$.

Nessa ótica, encontrou-se que $68 \%$ dos entrevistados da primeira série concordaram em que a disposição dos estudantes em pequenos grupos facilitava o aprendizado do conteúdo, com suas dúvidas sendo sanadas em grupo. Em relação à segunda série, 92\% concordaram, algo observado também na terceira $(88 \%)$ e na quarta série $(92 \%)$. Nota-se que há crescente valorização da importância da disposição em pequenos grupos. Com base em um estudo de revisão sobre o trabalho em pequenos grupos de $\operatorname{Costa}^{13}$, demonstra-se que esta estratégia tende a nutrir a dimensão social do estudante, potencializando sua criatividade, capacidade de resolução de problemas e desenvolvimento de pensamento crítico. Nessa perspectiva, as questões seguintes avaliaram a percepção dos estudantes sobre o trabalho em pequenos grupos e repercussões em sua aprendizagem.

Segundo alguns autores ${ }^{5}$, a cooperação pode promover habilidades importantes na formação do médico com melhor desenvoltura para questões psicossociais. Por isso, avaliou-se se havia ganhos em relação ao trabalho em equipe e relações interpessoais. No tocante ao primeiro tópico, $75 \%$ dos entrevistados da primeira série concordaram com a ideia, assim como $81 \%$ da segunda, $92 \%$ da terceira e 90\% da quarta série. Quando indagados sobre o segundo tópico (aprimoramento em relações interpessoais), 54\% dos estudantes da primeira série concordaram, assim como $56 \%$ da segunda, $63 \%$ da terceira e $73 \%$ da quarta série.

A Tabela 2 mostra o perfil dos docentes atuantes nas atividades teórico-práticas da primeira à quarta série do curso de Medicina da Famema, por graduação, tempo de formação, tempo de trabalho com metodologias ativas e pós-graduação. Tem-se que $85 \%$ desses docentes se graduaram em método tradicional, majoritariamente com 16 a 30 anos de formação, com 15 a 21 anos de trabalho com métodos ativos, e 92\% destes com pós-graduação.
TABELA 2

Caracterização sociodemográfica dos docentes da primeira à quarta série da Famema

$\begin{array}{lcc}\text { Variáveis } & \text { N } & \% \\ \text { Graduação } & & \\ \text { Método tradicional } & 82 & 85 \\ \text { Métodos ativos } & 15 & 15 \\ \text { Tempo de formação (anos) } & & \\ 1-15 & 16 & 16 \\ 16-30 & 46 & 47 \\ 31-45 & 35 & 36 \\ \text { Pós-graduação } & & \\ \text { Não } & 89 & 90 \\ \text { Sim } & 8 & 8 \\ \text { Tempo de trabalho com métodos ativos (anos) } & & \\ 1-7 & 13 & 13 \\ 8-14 & 25 & 26 \\ 15-21 & 59 & 61 \\ \text { Total } & \mathrm{N}=97 & \%=100\end{array}$

Fonte: Os autores, 2018.

Quando questionados se já haviam tido experiência com atuação em pequenos grupos antes de começar a trabalhar na Famema, 52\% afirmaram que sim. Sobre participação em atividades de capacitação para trabalhar com pequenos grupos inseridos em métodos ativos, 92\% afirmaram que sim. Quando interrogados se já haviam estudado aprendizagem cooperativa e colocado este conceito em prática, $78 \%$ afirmaram que sim.

Freitas et al. ${ }^{16}$ afirmam que, na sua prática, o professor necessita pensar sobre o processo educacional a fim de estruturá-lo, possibilitando aos acadêmicos mudar pensamentos, ações e condutas, bem como ampliar e solidificar sua aprendizagem. Autores reafirmam a necessidade de o professor desempenhar papel de liderança no grupo e estar treinado para seguir adequadamente a metodologia, estimulando o grupo mediante perguntas $^{17}$.

A segunda seção do questionário comparava metodologias ativas com o ensino tradicional em relação às suas vantagens.Assim, os docentes foram questionados se havia avanços em trabalho em equipe ( $88 \%$ concordaram) e também se percebiam benefícios em relações interpessoais (87\% concordaram) - concepções que corroboram aquelas dos estudantes.

No tocante à análise qualitativa, com base nas falas de estudantes e docentes, elencaram-se quatro categorias temáticas para análise:

- Abordagem conceitual;

- Benefícios da cooperação;

- Desenvolvimento mútuo de professores e estudantes por meio de cooperação;

- Avaliação e educação permanente dos professores. 


\section{Abordagem conceitual}

Este tópico determina o grau de aprofundamento de professores e acadêmicos em relação à aprendizagem cooperativa. Observou-se que, entre os entrevistados, uma parcela de professores e estudantes relatou nunca ter ouvido falar desta terminologia:

Não, não tenho nenhum conhecimento.(E1)

Aprendizagem cooperativa não, não conheço esse termo.(P2)

No entanto, alguns estudantes, apesar de não conhecerem exatamente o conceito, extraíram do termo o que acreditavam a ele corresponder. Nesse sentido, alguns docentes referiram que já o haviam estudado, como nas falas seguintes:

[...] seria mais ou menos o que a gente faz principalmente nas sessões de tutoria, a questão de compartilhar o conhecimento.

A cooperação no sentido de realmente estabelecer este trabalho em grupo, todos têm a responsabilidade com o aprendizado de todos. (E3)

Já, já li... Costumo pegar artigo na internet, nas bibliotecas médicas para ler alguma coisa nesse sentido. (P1)

Johnsonet al. ${ }^{7}$ propõem cinco componentes básicos para serem incorporados ao processo de ensino e aprendizagem para que haja aprendizagem cooperativa, tendo o professor como facilitador nesse processo. São eles: interdependência positiva, responsabilidade individual e grupal, interação face a face, habilidades interpessoais e grupais para manter um ambiente adequado ao aprendizado, e avaliação grupal.

Tolsgaardet al. ${ }^{18}$ relatam em seu projeto que, quando requisitos básicos para a cooperação são preenchidos em grupos de debate, há de fato aquisição de habilidades clínicas, psicomotoras, sociais e cognitivas. Portanto, para contemplar estes aspectos e propiciara construção do conhecimento, é necessário que todos os elementos do grupo conheçam de forma ampla o conceito de aprendizagem cooperativa e o seu valor.

\section{Benefícios da cooperação}

Vega Vacaet al. ${ }^{19}$ destacam em seu trabalho que a aprendizagem cooperativa traz vários benefícios num contexto de trabalho em pequenos grupos: aumenta o rendimento, motivação e autoestima dos estudantes frente à aprendizagem individualista, desenvolve atitudes mais positivas em relação à aprendi- zagem e aos docentes, favorece a aceitação dos companheiros e desenvolve interdependência positiva.

Os entrevistados desta pesquisa também citaram explicitamente citaram benefícios relacionados a domínios cognitivos, sociais e psicomotores, como exemplificado nos discursos a seguir:

[...] você aprende a falar em público, você desenvolve raciocínio junto com os outros, além de desenvolver um pouco da sua personalidade também, ajuda a ver onde você erra, os seus defeitos, que vão melhorando com o tempo [...

[...] o aprendizado cooperativo é isso, você tem uma habilidade, o outro tem outra, e vocês juntos podem pegar um pouco mais da habilidade do outro, do conhecimento do outro, da atitude do outro, e então forma-se uma competência melhor. (P6)

Quando você fala, você também aprende, porque você se esforçou pra entender pra poder explicar pro outro.(E8)

Nesse sentido, apesar de não terem formação sobre a psicologia da aprendizagem, especificamente sobre aprendizagem cooperativa, na prática reconhecem sua importância.

Desenvolvimento mútuo de estudantes e professores por meio de cooperação

No contexto de interação entre professores e acadêmicos nas atividades da graduação, estes nomearam vantagens que a cooperação mútua propicia um ao outro, demonstrando a possibilidade de aprendizado inclusive da parte dos docentes. Discursos que bem exemplificam este achado são:

[...] tive que ver na internet de onde ele tinha tirado aquilo, foi muito legal, porque eu também pude aprender, porque era uma coisa supernova, acho que era 2014, e era um trabalho de 2012 que eu não tinha visto. (P3)

[...] quando o tutor de fato cumpre a sua função como tutor, ele consegue estimular e fazer com que esse raciocínio clínico seja despertado.(E3)

Estas percepções de aprendizagem mútua entre professores e estudantes também são encontradas no estudo de Freitas et al. ${ }^{16}$, ao afirmarem que a relação existente entre eles permite a troca de saberes, sendo condição indispensável ao processo ensino-aprendizagem, o que dinamiza o processo educativo e lhe dásentido. Assim, nesta relação tutorial, o professor tam- 
bém experimenta mudanças, isto é, ao conhecer e se aproximar mais dos estudantes, pode rever suas ideias, aprimorar seu currículo e, sobretudo, refletir sobre seus valores e julgamentos ${ }^{20}$.

\section{Avaliação e educação permanente dos professores}

Estudantes e professores trouxeram a importância do cumprimento de determinadas competências por docentes para que o processo de ensino e aprendizagem se torne mais efetivo. Alguns acadêmicos relatam que é essencial que os professores tenham uma postura cooperativa com eles, sobretudo com aqueles que estão chegando ao primeiro anoe têm dificuldade para se adaptar:

Quando eu cheguei, eu senti falta de um apoio de professor, me sentia completamente abandonada.(E8)

Esta percepção de dificuldade de adaptação também está presente em estudo anterior que já demonstrava a necessidade de os estudantes receberem o apoio de professores para se adaptarem ao método ${ }^{21}$.

Docentes também trouxeram a importância de haver instrumentos capazes de avaliar sua prática a fim de propiciar capacitação continuada dos professores, para que atuem segundo as metodologias ativas de forma mais eficaz, como na fala:

Avaliar sempre quando vocês fazem esses trabalhos com relação ao método, eu acho muito significativo, para ver o quanto isso é efetivo, o quanto faz sentido. Mas tem que avaliar sempre, pra ir ajustando, porque às vezes desafina. (P4)

Ao questionar-se se haviam sido preparados para atuar em pequenos grupos segundo a proposta de metodologias ativas, docentes relataram que sim, de forma permanente na instituição, como no discurso que se segue:

Quando começou a UPP [Unidade de Prática Profissional] no formato de hoje, a gente teve uma capacitação prévia, mas a grande capacitação na minha opinião foi a educação permanente durante todo o curso. (P3)

Na Famema, a Educação Permanente foi instalada em 1997 juntamente com a reformulação do modelo educacional, que passou do tradicional a formas ativas ${ }^{22}$. Essas estratégias trabalham para a formação de grupo, para o trabalho em equipe e instrumentaliza docentes para o seu exercício ${ }^{23}$. Assim, os participantes desta pesquisa confirmam essas afirmações, o que expressa a necessidade de continuar a investir na sua capacitação, pois existe também a renovação do quadro de professores.

\section{CONSIDERAÇÕES FINAIS}

Este trabalho teve a aspiração de explorar concepções de docentes e estudantes a respeito da aprendizagem cooperativa vivenciada em pequenos grupos no âmbito de metodologias ativas de ensino e aprendizagem, além de promover a caracterização de seus perfis a fim de investigar os benefícios proporcionados pela cooperação. Identificar os anseios para adaptação da docência neste processo também foi uma meta.

Neste sentido, observou-se que os resultados quantitativos e qualitativos apontaram aspectos semelhantes. Os participantes da pesquisa apresentaram concepções análogas quanto aos benefícios da aprendizagem cooperativa em pequenos grupos, julgando que habilidades de comunicação, raciocínio crítico, interdependência positiva, avanços em trabalho em equipe, além de boa aquisição de conhecimento cognitivo são garantidos neste processo.

Observou-se também que, à medida que avançavam nas séries, os estudantes apresentavam percepções um pouco diferentes em relação a diversas questões. Este fato talvez esteja relacionado à maturidade advinda com a própria idade e principalmente a partir da experiência com os métodos ativos, como evidenciado nas maiores porcentagens descritas da quarta série quando questionados se percebiam mais cooperação do que competição em pequenos grupos.

Em suma, com base no entendimento de docentes e estudantes, concluiu-se que a aprendizagem cooperativa é eficaz em pequenos grupos e que traz benefícios sociais, cognitivos e psicomotores, sendo favorecida no âmbito de metodologias ativas e com a educação permanente, a qual, se implementada de maneira incisiva, pode gerar ferramentas úteis aoprocesso de ensino e aprendizagem.

Notou-se que o tema aprendizagem cooperativa ainda não foi muito explorado na literatura da área médica, talvez por estar de certa maneira implícito no domínio de metodologias ativas. Os resultados encontrados neste estudo, cujo desenvolvimento ocorreu numa instituição que tem utilizado métodos ativos nos últimos 20 anos, poderiam ser úteis para outras escolas médicas que almejam adotar ou já adotaram a metodologiamais recentemente, com a finalidade de orientar gestores e coordenadores do curso quanto à importância da aprendizagem por cooperação.

Propõe-se realizar novos trabalhos com este enfoque a fim de confirmar os resultados encontrados nesta pesquisa, assim como expandir os aspectos da aprendizagem cooperativa em pequenos grupos relacionada a metodologias ativas no espectro da educação médica. 


\section{AGRADECIMENTOS}

Ao incentivo e apoio financeiro recebidos do Programa Institucional de Bolsa de Iniciação Científica (Pibic) e do Conselho Nacional de Desenvolvimento Científico e Tecnológico (CNPq).

\section{REFERÊNCIAS}

1. Brasil. Ministério da Educação. Conselho Nacional de Educação. Câmara de Educação Superior. Resolução CNE/CES no 3, de 20 de junho de 2014. Institui diretrizes curriculares nacionais do curso de graduação em medicina e dá outras providências. Diário Oficial da União. Brasília, 23 jun. 2014; Seção 1, p. 8.

2. Mello CCB, Alves RO, Lemos SMA. Metodologias de ensino e formação na área da saúde: revisão de literatura. Rev CEFAC 2014;16(6):2015-28.

3. Barreto NAP, Xavier AREO, Sonzogno MC. Percepção de tutores quanto a sua avaliação pelos discentes de um curso médico. Rev Bras Educ Méd 2017;41(2):221-30.

4. Damiani MF. Entendendo o trabalho colaborativo em educação e revelando seus benefícios. Educ Rev 2008(31):213-30.

5. Pinho EM, Ferreira CA, Lopes JP. As opiniões de professores sobre a aprendizagem cooperativa. Rev Diálog Educ 2013;13(40):913-37.

6. Piaget J. Estudos sociológicos. São Paulo: Companhia Editora Forense; 1973.

7. Johnson DW, Jonhson RT, Holubec EJ.El aprendizaje cooperativo en el aula. Buenos Aires: Paidós; 1999.

8. Almeida MTC, Batista NA. Ser docente em métodos ativos de ensino-aprendizagem na formação do médico. Rev Bras Educ Méd 2011;35(4):468-76.

9. Bolfarine H, Bussab WO. Elementos de amostragem. 3a reimp. São Paulo: Edgard Blücher; 2012.

10. Fontanella BJB, Ricas J, Turato ER. Amostragem por saturação em pesquisas qualitativas em saúde: contribuições teóricas. Cad Saúde Pública2008;24(1):17-27.

11. Minayo MCS. O desafio do conhecimento: pesquisa qualitativa em saúde. 13a ed. São Paulo: Hucitec; 2013.

12. Gomes R. Análise e interpretação de dados de pesquisa qualitativa. In: Minayo MCS, org. Pesquisa social:teoria, método e criatividade. 32a ed. Petrópolis: Vozes; 2012. p. 79-108.

13. Botti SHO, Rego S. Preceptor, supervisor, tutor e mentor: quais são seus papéis? Rev Bras Educ Méd 2008;32(3):363-73.

14. Costa MCG, Mazzoni CJ, Braccialli LAD, Moraes MAA. Exercício de avaliação da prática profissional como estratégia de ensino e aprendizagem.Avaliação (Campinas) 2011;16(3):675-84.
15. Albanese M. Problem-based learning: why curricula are likely to show little effect on knowledge and clinical skills. Med Educ 2000;34(9):729-38.

16. Freitas DA, Santos SEM, Lima LVS, Miranda LN, Vasconcelos EL, Nagliate PC. Saberes docentes sobre processo ensino-aprendizagem e sua importância para a formação profissional em saúde. Interface (Botucatu) 2016;20(57):437-48.

17. Moreira MB,Manfroi WO.O papel da aprendizagem baseada em problemas nas mudanças no ensino médico no Brasil. Clin Biomed Res 2011;31(4):477-81.

18. Tolsgaard MG,Kulasegaram KK,Ringsted CV. Collaborative learning of clinical skills in health professions education: the why, how, when and for whom. Med Educ 2016;50(1):69-78.

19. Vega Vaca ML, Vidal Rodriguez D, Del Pilar Garcia M. Avances acerca de los efectos del aprendizaje cooperativo sobre el logro académico y las habilidades sociales en relación com el estilo cognitivo. Rev ColombEduc 2013;64:155-74.

20. Chaves LJ, Gonçalves ECQ, Ladeira LR, Ribeiro MS, Costa MB, Ramos AAM. A tutoria como estratégia educacional no ensino médico. Rev Bras Educ Med 2014;38(4):532-41.

21. Moraes MAA, Manzini EJ. Concepções sobre a aprendizagem baseada em problemas: um estudo de caso na Famema. Rev Bras Educ Med 2006;30(3):125-35.

22. Nunes CRR, Rolin LMG, Lopes VMM. Os primórdios da educação permanente na academia e nos serviços de saúde. In: Frascischetti I, Colela ACM, Vieira CM, Lazarini CA, Pio DAM, Rolin LMG, et al., org. Educação permanente da academia: da teoria à prática. Curitiba: CRV; 2014. p. 17-27.

23. Viera CM, Soares MOM, Locatelli RR. Educação permanente na academia (EPA): fundamentos, planejamento e estratégias. In: Frascischetti I, Colela ACM, Vieira CM, Lazarini CA, Pio DAM, Rolin LMG, et al., org. Educação permanente da academia: da teoria à prática. Curitiba: CRV; 2014. p. 37-48.

\section{CONTRIBUIÇÃO DOS AUTORES}

Todos contribuiram igualmente

\section{CONFLITO DE INTERESSES}

Não há

\section{ENDEREÇO PARA CORRESPONDÊNCIA}

Faculdade de Medicina de Marilia

Avenida José de Grande 332 Jardim Parati,

Marilia 17519-030, Brasil unrestricted use, distribution, and reproduction in any medium, provided the original work is properly cited. 